\title{
The analysis of multifactor experiments designed to contrast the within- and between-subjects manipulation of the independent variables
}

\author{
ALBERT ERLEBACHER \\ Northwestern University, Evanston, Illinois 60201
}

\begin{abstract}
Experiments are frequently performed in which the major question of interest is whether the effect of the substantive independent variable depends on its manipulation within as opposed to between subjects. Erlebacher (1977) discussed the design of such experiments in which one independent variable is manipulated, and presented a method of analysis by an analysis of variance technique. This paper discusses the analysis of three different experimental designs in which the within-subjects/between-subjects design type is one factor and two substantive independent variables are manipulated. A rule is also presented that can be used to generate statistical tests in a generalized multifactor design.
\end{abstract}

Within- and between-subjects designs of experiments have often been used interchangeably in psychology. Many experiments, though, have shown that the two design types sometimes yield different results. Erlebacher (1977) listed a number of experiments in several different areas of psychology that were designed specifically to investigate the designtype effect. Typically, in such an experiment, one has a substantive independent variable which is varied between groups. One additional group of subjects receives all the levels of the independent variable. The major question of concern is whether the difference in means among the conditions in the betweensubjects design is equal to the difference in means among the conditions in the within-subjects design. If we explicitly designate design type as a second independent variable, we may phrase the major question of concern as whether there exists an interaction between the substantive independent variable and the design type.

Erlebacher (1977) presented an analysis of variance procedure for analyzing the results of experiments in which the effect of one substantive independent variable and its interaction with design type is investigated. This paper presents the appropriate analysis of variance procedure for three experimental design cases in which there exist two substantive independent variables in addition to the design variable. The pro-

Requests for reprints should be sent to Albert Erlebacher, Department of Psychology, Northwestern University, Evanston, Illinois 60201 . cedures may be generalized to the case of three or more substantive independent variables.

\section{THE THREE CASES}

In all three cases there are two substantive independent variables, $\mathrm{A}$ and $\mathrm{B}$. Let $\mathrm{A}$ have I levels and let there be $J$ levels of variable $B$. The design factor is designated $D$. In each case there are two levels of the design factor. These are designated $D_{1}$ and $D_{2}{ }^{1}$ In the first case, variable $B$ is manipulated between subjects. Variable A, however, is a within-subjects variable at $D_{1}$ but a between-subjects variable at $D_{2}$. We designate this as Case $\mathrm{AwBb}-\mathrm{AbBb}$. In Case $\mathrm{AwBw}-\mathrm{AbBw}$, variable $\mathrm{B}$ is always manipulated within subjects, but variable $A$ is a within-subjects variable at $D_{1}$ and a between-subjects variable at $D_{2}$. Finally, in Case $\mathrm{AwBw}-\mathrm{AbBb}$, both variables are withinsubjects variables at $D_{1}$ but between-subjects at $D_{2}$. Let there be $n$ subjects in each of the independent groups.

\section{Case 1: AwBb-AbBb}

In the first case, the experiment consists of $\mathrm{J}(\mathrm{I}+1)$ independent groups and, therefore, $\mathrm{nJ}(\mathrm{I}+1)$ subjects. At $D_{1}$, there are $J$ groups. These groups are treated according to the different levels of variable B. Each of the $n J$ subjects at $D_{1}$, however, receives all $I$ treatments of variable $A$. At $D_{2}$, there are IJ independent groups and, therefore, nIJ subjects. Each of these groups receives one of the $I J$ treatments resulting from the $I \times J$ crossing of variables $A$ and $B$.

The additive score model has $\mathrm{X}_{\mathrm{ijks}}$, the score of 
subject $s$ in design type $k$ and in levels $i$ and $j$ of variables $A$ and $B$, respectively. We have

$$
\begin{aligned}
\mathrm{X}_{\mathrm{ijks}}= & \mu \ldots+\alpha_{\mathrm{i}} \ldots+\beta_{. \mathrm{j}} .+\delta_{\ldots \mathrm{k}} \\
& +(\alpha \beta)_{\mathrm{ij}} . .+(\alpha \delta)_{\mathrm{i} . \mathrm{k} .}+(\beta \delta)_{. \mathrm{jk}} . \\
& +(\alpha \beta \delta)_{\mathrm{ijk} .}+\pi_{\mathrm{ijks}}+(\alpha \pi)_{\mathrm{ijks}} \\
& +\varepsilon_{\mathrm{ijks}},
\end{aligned}
$$

where $\mu \ldots$ is a common value that is part of all scores; $\alpha_{\mathrm{i}} \ldots$ is the contribution of variable $A$ in its ith level (let $\mathrm{i}=1,2, \cdots, \mathrm{I}) ; \beta . j$ is the contribution of variable $B$ in the $j$ th level (let $\mathrm{j}=1,2, \cdots, \mathrm{J}$ ); $\delta . \mathbf{k}$. is the contribution of the design type $(k=$ $1,2) ;$ the next four terms represent the contributions of the first-and second-order interactions among the three independent variables; $\pi_{\mathrm{ijks}}$ is the contribution due to subject $\mathrm{s}$ in condition ijk (there are $\mathrm{n}$ subjects in each of the conditions, i.e., $s=1,2, \cdots, n$ ); $(\alpha \pi)_{\text {ijks }}$ represents the interaction of subjects with independent variable $A ; \varepsilon_{i j k s}$ is random error.

The design type, $D$, and the substantive independent variables, $A$ and $B$, are considered to be fixed variables. Subjects is a random variable. The usual analysis of variance assumptions are made, with the exception that, in design-type $D_{1}$,

$$
\sum_{\mathrm{i}}(\alpha \pi)_{\mathrm{ij} 1 \mathrm{~s}}=0,
$$

while, in design-type $D_{2}$,

$$
E(\alpha \pi)_{i j 2 s}=0
$$

and

$$
\mathrm{E}(\alpha \pi)_{\mathrm{ijks}}^{2}=\sigma_{\mathrm{As}}^{2},
$$

E indicating expected value.

The total sum of squares should be partitioned according to the sources of variation presented in Table 1. The table also indicates the degrees of freedom and the definitional formulas for the sums of squares.

The means needed for the sums of squares can be expressed in terms of the effects from the model. This is done in Table 2. For purposes of arriving at

\begin{tabular}{|c|c|c|}
\hline Source of Variation & $\mathrm{df}$ & Sum of Squares \\
\hline A & $I-1$ & $2 \operatorname{Jn} \Sigma\left(\bar{X}_{i} \ldots-\bar{X}_{\ldots} \ldots\right)^{2}$ \\
\hline B & $\mathrm{J}-\mathbf{1}$ & $\left.\underset{j}{2 \ln \Sigma\left(\bar{X}_{. j} .\right.}-\bar{x}_{\ldots}\right)^{2}$ \\
\hline$A \times B$ & $(I-1)(J-1)$ & $2 n \sum \sum\left(\bar{x}_{i j} \ldots-\bar{x}_{i} \ldots-\bar{x}_{. j}+\bar{x}_{\ldots} \ldots\right)^{2}$ \\
\hline Design Type (D) & 1 & $\operatorname{IJn} \Sigma_{k}\left(\bar{X}_{\ldots k}-\bar{X}_{\ldots}\right)^{2}$ \\
\hline$A \times D$ & $I-1$ & $\operatorname{Jn} \sum \Sigma k_{i k} \bar{X}_{i, k}-\bar{X}_{i \ldots}-\bar{X}_{\ldots k}+\bar{X}_{\ldots)^{2}}$ \\
\hline$B \times D$ & $\mathbf{J}-1$ & 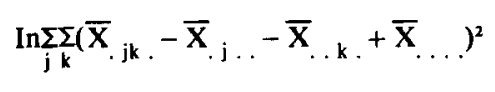 \\
\hline$A \times B \times D$ & $(\mathrm{I}-1)(\mathrm{J}-1)$ & $\begin{array}{l}n \sum_{i j k} \sum \bar{X}_{i j k}-\bar{X}_{i j \ldots}-\bar{X}_{i \cdot k}-\bar{X}_{. j k} \\
+\bar{X}_{i \ldots}+\bar{X}_{. j \ldots}+\bar{X}_{\ldots k}-\bar{X}_{\ldots)^{2}}\end{array}$ \\
\hline Subjects $/ B\left(D_{1}\right.$ only) $\left[s / B\left(D_{1}\right)\right]$ & $\mathbf{J}(\mathbf{n}-1)$ & $\left.I_{j s} \sum \Sigma_{. j 1 s}-\bar{X}_{. j 1}\right)^{2}$ \\
\hline$A \times s / B\left(D_{1}\right)$ & $\mathbf{J}(\mathbf{l}-1)(\mathrm{n}-1)$ & $\left.\sum_{i j s} \sum X_{i j 1 s}-\bar{X}_{i j 1}-\bar{X}_{j j 1 s}+\bar{X}_{. j 1}\right)^{2}$ \\
\hline $\mathrm{s} / \mathrm{AB}\left(\mathrm{D}_{2}\right)$ & $1 J(n-1)$ & $\left.\sum_{\mathrm{i} j \mathrm{~s}} \sum_{\mathrm{ij} 2 \mathrm{~s}}-\overline{\mathrm{X}}_{\mathrm{ij2} 2}\right)^{2}$ \\
\hline Total & $2 \mathrm{IJn}-1$ & $\left.\sum_{i j k s} \sum_{j=1} x_{i j k s}-\bar{x}_{\ldots}\right)^{2}$ \\
\hline
\end{tabular}
the expected values of mean squares, note that in $D_{1}$, since the same subjects are measured in all levels of A, $\pi_{\text {.jls }}$ is based on but one score, $\pi_{. i 1}$, is based on $n$ scores and $\pi \ldots 1$ is based on Jn scores. This

Table 1

Partitioning of the Total Sum of Squares for Case 1 
Table 2

Means Expressed in Terms of Model Effects: Case 1

\begin{tabular}{|c|c|}
\hline Mean & Expression in Effects \\
\hline$\overline{\mathrm{X}}$ & $\mu \ldots+\frac{\bar{\pi} \ldots 1}{2}+\frac{\bar{\pi} \ldots 2}{2}+\frac{(\overline{\alpha \pi}) \ldots 2}{2}+\bar{\varepsilon}$ \\
\hline $\bar{x}_{i}$ & $\mu \ldots+\alpha_{\mathrm{i}} \ldots+\frac{\bar{\pi} .1}{2}+\frac{\bar{\pi}_{\mathrm{i}} .2}{2}+\frac{(\overline{\alpha \pi})_{\mathrm{i}} 1}{2}+\frac{(\overline{\alpha \pi})_{\mathrm{i} .2}}{2}+\bar{\varepsilon}_{\mathrm{i}}$ \\
\hline $\bar{X}_{. j}$ & $\mu \ldots+\beta_{. j}+\frac{\bar{\pi}_{. j 1}}{2}+\frac{\bar{\pi}_{i 2}}{2}+\frac{(\overline{\alpha \pi})_{. j 2}}{2}+\bar{\varepsilon}_{. j} \ldots$ \\
\hline$\overline{\mathrm{X}}_{\mathrm{ij}}$ & $\mu \ldots+\alpha_{i} \ldots+\beta_{. j} .+(\alpha \beta)_{i j} \ldots+\frac{\bar{\pi}_{j 1}}{2}+\frac{\bar{\pi}_{i j 2}}{2}+\frac{(\overline{\alpha \pi})_{i j 1}}{2}+\frac{(\alpha \pi)_{i j 2}}{2}+\bar{\varepsilon}_{i j} \ldots$ \\
\hline$\overline{\mathrm{x}} \ldots$ & $\mu_{\ldots}+\delta_{\ldots 1}+\bar{\pi}_{11}+\bar{\varepsilon}_{11}$ \\
\hline$\overline{\mathrm{X}}, 2$ & $\mu_{\ldots}+\delta_{, 2}+\bar{\pi}, 2+(\overline{\alpha \pi})_{\ldots 2}+\bar{\varepsilon}_{, 2}$ \\
\hline$\overline{\mathbf{X}}_{\mathbf{i} .1}$ & $\mu_{\ldots}+\alpha_{i \ldots}+\delta_{\ldots 1}+(\alpha \delta)_{i, 1}+\bar{\pi}_{1.1}+(\overline{\alpha \pi})_{i, 1}+\bar{\varepsilon}_{i, 1}$ \\
\hline $\bar{X}_{i .2 .}$ & $\mu_{\ldots}+\alpha_{\mathrm{i}, \ldots}+\delta_{\ldots 2}+(\alpha \delta)_{\mathrm{i}, 2}+\bar{\pi}_{\mathrm{i}, 2}+(\overline{\alpha \pi})_{\mathrm{i}, 2 .}+\bar{\varepsilon}_{\mathrm{i}, 2}$ \\
\hline$\overline{\mathbf{X}}_{. \mathrm{j} 1}$ & $\mu_{\ldots}+\beta_{. j}+\delta_{\ldots 1}+(\beta \delta)_{. j 1}+\bar{\pi}_{\mathrm{j} 1}+\bar{\varepsilon}_{\mathrm{j} 1}$ \\
\hline $\bar{X}_{. j 2}$ & $\mu_{\ldots}+\beta_{. j \ldots}+\delta_{.2 .}+(\beta \delta)_{. j 2}+\bar{\pi}_{. j 2}+(\overline{\alpha \pi})_{. j 2}+\bar{\varepsilon}_{\mathrm{j} 2}$ \\
\hline$\overline{\mathrm{X}}_{\mathrm{ij} 1}$. & 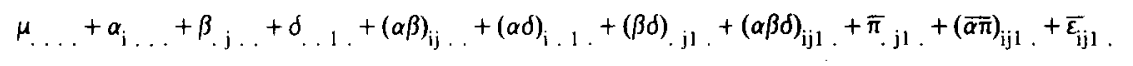 \\
\hline$\overline{\mathrm{X}}_{\mathrm{ij} 2}$ & $\mu_{\ldots}+\alpha_{\mathrm{i}} \ldots+\beta_{\mathrm{j}, \ldots}+\delta_{\ldots 2}+(\alpha \beta)_{\mathrm{ij}}+(\alpha \delta)_{\mathrm{i} .2}+(\beta \delta)_{\mathrm{j} 2}+(\alpha \beta \delta)_{\mathrm{ij} 2}+\bar{\pi}_{\mathrm{ij} 2}+(\overline{\alpha \pi})_{\mathrm{ij} 2}+\bar{\varepsilon}_{\mathrm{ij} 2}$ \\
\hline$\overline{\mathrm{X}}_{. \mathrm{jls}}$ & 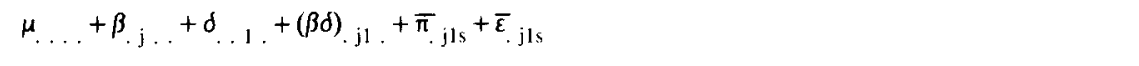 \\
\hline$X_{i j 1 s}$ & $\begin{array}{l}\mu_{\ldots} \ldots+\alpha_{\mathrm{i}} \ldots+\beta_{. \mathrm{j}}+\delta_{\ldots 1}+(\alpha \beta)_{\mathrm{ij}} \ldots+(\alpha \delta)_{\mathrm{i}, 1}+(\beta \delta)_{. \mathrm{j} 1}+(\alpha \beta \delta)_{\mathrm{ij} 1}+\pi_{. \mathrm{j} 1 \mathrm{~s}}+(\alpha \pi)_{\mathrm{ij} 1 \mathrm{~s}} \\
+\varepsilon_{\mathrm{ijl} 1 \mathrm{~s}}\end{array}$ \\
\hline
\end{tabular}

differs from the analogous values in $D_{2}$, which are all based on I times that many scores. This fact has implications for the values of variances based on these terms.

The expected values of the mean squares associated with each of the sources of variation are given in Table 3. The $\mathrm{S}^{2}$ refer to "variances" for mixed and fixed effects, while the $\sigma^{2}$ are true variances for purely random effects. The subscripts refer to the effects due to the sources of variation. Double or triple subscripts indicate a variance due to the effect of an interaction. The final value in each line, $\sigma_{\varepsilon}^{2}$, refers to error variance.

The expected values of the mean squares indicate that, in order to make a statistical test of the effectiveness of any potential source of variation, one must form "quasi-F"' ratios by dividing the mean square for the effect being tested by a linear combination of mean squares. Two different denominators need to be used. To test the effect of $A, A \times B, A \times D$, and $A \times B \times D$, the denominator for the quasi-F ratio is

Denominator $\left.1 \mathrm{a}=\left[\mathrm{MS}_{\mathrm{Axs} / \mathrm{B}\left(\mathrm{D}_{1}\right)}+\mathrm{MS}_{\mathrm{S} / \mathrm{AB}\left(\mathrm{D}_{2}\right)}\right)\right] / 2$.
For testing the effect of $\mathrm{B}, \mathrm{D}$, and $\mathrm{B} \times \mathrm{D}$, one uses

$$
\text { Denominator } 1 \mathrm{~b}=\left[\mathrm{MS}_{\mathrm{S} / \mathrm{B}\left(\mathrm{D}_{1}\right)}+\mathrm{MS}_{\mathrm{s} / \mathrm{AB}\left(\mathrm{D}_{2}\right)}\right] / 2 \text {. }
$$

The last column of Table 3 indicates which of the two denominators is to be used for each of the ratios. The degrees of freedom associated with each of the denominators must be approximated. Satterthwaite's (1946) formula can be used. His procedure determines that the number of degrees of freedom, $v$, associated with the sum (or mean) of two mean squares, $\mathrm{MS}_{1}$ and $\mathrm{MS}_{2}$, with associated degrees of freedom $v_{1}$ and $v_{2}$, respectively, is

$$
\left.v=\left(\mathrm{MS}_{1}+\mathrm{MS}_{2}\right)^{2} /\left[\mathrm{MS}_{1}^{2} / v_{1}\right)+\left(\mathrm{MS}_{2}^{2} / v_{2}\right)\right]
$$

\section{Case 2: AwBw-AbBw}

In the second case, the experiment has $I+1$ independent groups and, hence, $\mathrm{n}(\mathrm{I}+1)$ subjects. In design $D_{1}$, there is but a single group. This group receives all $I J$ treatments resulting from the $I \times J$ crossing of variables $A$ and $B$. In design $D_{2}$, there are I independent groups. Each is treated according to 
Table 3

Expected Values of Mean Squares for Case 1

\begin{tabular}{|c|c|c|}
\hline Source of Variation & Expected Value of Mean Square & Denominator \\
\hline A & $2 \mathrm{JnS}_{\mathrm{A}}^{2}+\frac{1}{2} \sigma_{\mathrm{S}}^{2}+\frac{(2 \mathrm{I}-1)}{2 \mathrm{I}} \mathrm{S}_{\mathrm{AS}}^{2}+\sigma_{\varepsilon}^{2}$ & $1 \mathrm{a}$ \\
\hline B & $2 \operatorname{InS} S_{B}^{2}+\frac{(I+1)}{2} \sigma_{S}^{2}+\frac{(I-1)}{2 I} S_{A s}^{2}+\sigma_{\varepsilon}^{2}$ & lb \\
\hline $\mathbf{A} \times \mathbf{B}$ & $2 \mathrm{nS}_{\mathrm{AB}}^{2}+\frac{1}{2} \sigma_{\mathrm{S}}^{2}+\frac{(2 \mathrm{I}-1)}{2 \mathrm{I}} \mathrm{S}_{\mathrm{As}}^{2}+\sigma_{\varepsilon}^{2}$ & la \\
\hline D & $\mathrm{IJnS}_{\mathrm{D}}^{2}+\frac{(\mathrm{I}+1)}{2} \sigma_{\mathrm{s}}^{2}+\frac{(\mathrm{I}-1)}{2 \mathrm{I}} \mathrm{S}_{\mathrm{As}}^{2}+\sigma_{\varepsilon}^{2}$ & $1 b$ \\
\hline$A \times D$ & $\mathrm{JnS}_{\mathrm{AD}}^{2}+\frac{1}{2} \sigma_{\mathrm{S}}^{2}+\frac{(2 \mathrm{I}-1)}{2 \mathrm{I}} \mathrm{S}_{\mathrm{AS}}^{2}+\sigma_{\varepsilon}^{2}$ & $1 \mathrm{a}$ \\
\hline $\mathrm{B} \times \mathrm{D}$ & $\operatorname{InS}_{\mathrm{BD}}^{2}+\frac{(I+1)}{2} \sigma_{\mathrm{S}}^{2}+\frac{(I-1)}{2 I} S_{\mathrm{AS}}^{2}+\sigma_{\varepsilon}^{2}$ & $1 b$ \\
\hline$A \times B \times D$ & $\mathrm{nS}_{\mathrm{ABD}}^{2}+\frac{1}{2} \sigma_{\mathrm{S}}^{2}+\frac{(2 I-1)}{2 I} \mathrm{~S}_{\mathrm{AS}}^{2}+\sigma_{\varepsilon}^{2}$ & la \\
\hline $\mathrm{s} / \mathrm{B}\left(\mathrm{D}_{1}\right)$ & $\mathrm{I} \sigma_{s}^{2}+\sigma_{\varepsilon}^{2}$ & \\
\hline $\mathrm{Axs} / \mathrm{B}\left(\mathrm{D}_{1}\right)$ & $\mathrm{S}_{\mathrm{AS}}^{2}+\sigma_{\varepsilon}^{2}$ & \\
\hline $\mathrm{s} / \mathrm{AB}\left(\mathrm{D}_{2}\right)$ & $\sigma_{s}^{2}+\frac{(I-1)}{I} S_{A s}^{2}+\sigma_{\varepsilon}^{2}$ & \\
\hline
\end{tabular}

one of the I levels of variable A. All subjects, however, receive all of the $\mathrm{J}$ treatments of variable $\mathrm{B}$.

The model is slightly different from Case 1 . Here we have

$$
\begin{aligned}
\mathrm{X}_{\mathrm{ijks}}= & \mu \ldots+\alpha_{\mathrm{i}} \ldots+\beta_{. \mathrm{j} . .}+\delta_{. . \mathrm{k}} \\
& +(\alpha \beta)_{\mathrm{ij} .}+(\alpha \delta)_{\mathrm{i} . \mathrm{k} .}+(\beta \delta)_{. \mathrm{jk}} \\
& +(\alpha \beta \delta)_{\mathrm{ijk} .}+\pi_{\mathrm{i} . \mathrm{ks}}+(\alpha \pi)_{\mathrm{i} . \mathrm{ks}} \\
& +(\beta \pi)_{\mathrm{ijks}}+(\alpha \beta \pi)_{\mathrm{ijks}}+\varepsilon_{\mathrm{ijks}} .
\end{aligned}
$$

The first eight terms on the right are exactly the same as those in Case 1. The next two terms have a dot replacing the $\mathrm{j}$. The next two terms are new and are due to the $\mathrm{B} \times$ Subject and the $\mathrm{A} \times \mathrm{B} \times$ Subject interactions. The last term is due to random error. Other than the common analysis of variance assumptions, here we assume that in $D_{1}$

$$
\sum_{i}(\alpha \pi)_{i .1 s}=\Sigma(\alpha \beta \pi)_{i j 1 s}=0,
$$

while in $D_{2}$

$$
\mathrm{E}(\alpha \pi)_{\mathrm{i} .2 \mathrm{~s}}=\mathrm{E}(\alpha \beta \pi)_{\mathrm{ij} 2 \mathrm{~s}}=0
$$

and

$$
\begin{gathered}
\mathrm{E}(\alpha \pi)_{\mathrm{i}, \mathrm{ks}}^{2}=\sigma_{\mathrm{As}}^{2} \\
\mathrm{E}(\alpha \beta \pi)_{\mathrm{ijks}}^{2}=\sigma_{\mathrm{ABs}}^{2} .
\end{gathered}
$$

Table 4 indicates the Case 2 sources of variation in the partitioning of the total sum of squares, the associated degrees of freedom, and the definitional formulas for the sums of squares. Note that the first seven sources of variation and their sums of squares are unchanged from Case 1 .

Table 5 indicates the expression of the means needed for the sums of squares in terms of the model effects. Means with expressions unchanged from Case 1 are not listed. In order to arrive at expected values of mean squares, we must note differences in the number of independent scores going into the means of the two design types. In $D_{1}, \pi_{2}$ is is based on one score, $\bar{\pi}_{.1}$. is based on $n$ scores, $(\beta \pi)$. j1s is based on one score, and $(\overline{\beta \pi})$. $\mathrm{j} 1$. is based on n scores, whereas the analogous terms in $D_{2}$ are based on $I$ times that many scores.

Table 6 shows the expected values of the mean squares associated with each source of variation. Again, quasi-F ratios are needed in order to make statistical tests. Four different denominators are 
Table 4

Partitioning of the Total Sum of Squares for Case 2

Source of Variation

$A, B, A \times B, D, A \times D, B \times D$,

$A \times B \times D$

Subjects $\left(D_{1}\right.$ only) $\left[s\left(D_{1}\right)\right]$

$\operatorname{Axs}\left(D_{1}\right)$

$\operatorname{Bxs}\left(\mathrm{D}_{1}\right)$

$\operatorname{AxBxs}\left(\mathrm{D}_{1}\right)$

$\mathrm{s} / \mathrm{A}\left(\mathrm{D}_{2}\right.$ only) $\left[\mathrm{s} / \mathrm{A}\left(\mathrm{D}_{2}\right)\right]$

$\mathrm{Bxs} / \mathrm{A}\left(\mathrm{D}_{2}\right)$

Total
Sum of Squares

Same as Case 1

Same as Case 1

$n-1$

$\mathbb{I J}(\overline{\mathrm{X}} \ldots,-\overline{\mathrm{X}}, \ldots)^{2}$

$(\mathrm{I}-1)(\mathrm{n}-1)$

$\underset{i s}{J} \sum_{s}\left(\bar{X}_{i, 1 s}-\bar{X}_{i .1}-\bar{X} \ldots 1 s+\bar{X} \ldots\right)^{2}$

$(J-1)(n-1)$

$\operatorname{I\Sigma \Sigma }_{j \leq}\left(\bar{X}_{. j 1}-\bar{X}_{. j 1}-\bar{X}_{\ldots 1 s}+\bar{X}_{\ldots 1}\right)^{2}$

$(\mathrm{I}-1)(\mathrm{J}-1)(\mathrm{n}-1)$

$\sum_{i j s}^{\sum \sum \Sigma\left(X_{i j l s}\right.}-\bar{X}_{i j 1}-\bar{X}_{i .1 s}-\bar{X}_{. j 1 s}$

$+\bar{X}_{i, 1}+\bar{X}_{. j 1}+\bar{X}_{\ldots 1 s}-\bar{X}_{\ldots 1 .)^{2}}$

I $(\mathrm{n}-1)$

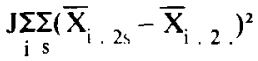

$\mathbf{I}(J-1)(n-1)$

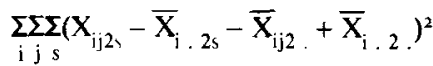

$\left.\sum_{i j k s} \sum \sum X_{i j k}-\bar{X} \ldots\right)^{2}$

Table 5

Means Expressed in Terms of Model Effects: Case 2

\section{Mean}

Expression in Effects

$\overline{\mathrm{x}}_{.}$

$$
\mu_{\ldots}+\beta_{. j}+\frac{\bar{\pi}_{.1}}{2}+\frac{\bar{\pi} .22}{2}+\frac{(\overline{\alpha \pi}) .2}{2}+\frac{(\overline{\beta \pi}) . j 1}{2}+\frac{(\overline{\beta \pi}) . j 2 .}{2}+\frac{(\overline{\alpha \beta \pi}) . j 2}{2}+\bar{\varepsilon}_{. j} .
$$

$\overline{\mathbf{X}}_{\mathrm{ij}}$

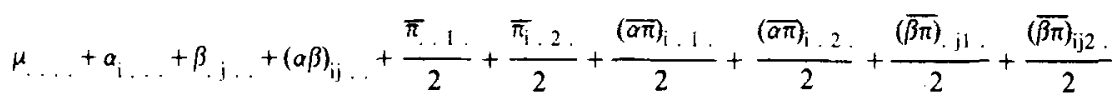

$+\frac{(\alpha \beta \pi)_{i j 1}}{2}+\frac{(\alpha \beta \pi)_{i j 2}}{2}+\bar{\varepsilon}_{i j}$

$\bar{X}_{j, j}$

$\mu_{\ldots}+\beta_{. j}+\delta_{\ldots 1}+(\beta \delta)_{. j 1}+\bar{\pi}_{\ldots 1}+\left(\overline{\beta \pi}_{. j 1}+\bar{\varepsilon}_{. j 1}\right.$.

$\overline{\mathbf{X}}_{. \mathrm{j} 2}$.

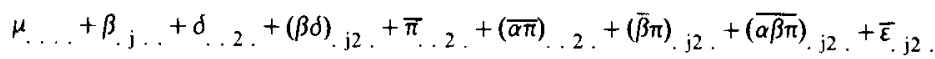

$\overline{\mathrm{X}}_{\mathrm{ij} 1}$.

$\mu_{\ldots}+\alpha_{i} \ldots+\beta_{. j}+d_{\ldots 1}+(\alpha \beta)_{i j}+(\alpha d)_{i, 1}+(\beta \delta)_{j 1}+(\alpha \beta d)_{i j 1}+\bar{\pi}_{.1}+(\overline{\alpha \pi})_{i .1}+(\overline{\beta \pi})_{j 1}$ $+\left(\overline{\alpha \beta \pi}_{\mathrm{ij1} 1}+\overline{\varepsilon_{\mathrm{ij} 1}}\right.$.

$\overline{\mathrm{X}}_{\mathrm{ij} 2}$.

$\mu_{\ldots}+\alpha_{\mathrm{i}} \ldots+\beta_{. \mathrm{j} . .}+\delta_{.2}+(\alpha \beta)_{\mathrm{ij}} \ldots+(\alpha \delta)_{\mathrm{i} .2}+(\beta \delta)_{. \mathrm{j} 2}+(\alpha \beta \delta)_{\mathrm{ij} 2}+\bar{\pi}_{\mathrm{i} .2}+(\overline{\alpha \bar{\pi}})_{\mathrm{i} .2 .}+(\overline{\beta \pi})_{\mathrm{ij} 2}$

$+(\overline{\alpha \beta \pi})_{i \mathrm{ij} 2}+\bar{\varepsilon}_{\mathrm{ij} 2}$.

$\overline{\mathrm{X}}_{\ldots 1 \mathrm{~s}} \quad \mu \ldots+\delta_{\ldots 1}+\pi_{\ldots 1 \mathrm{~s}}+\bar{\varepsilon} \ldots 1 \mathrm{~s}$

$\overline{\mathrm{X}}_{\mathrm{i}, 1 \mathrm{~s}} \quad \mu \ldots+\alpha_{\mathrm{i} \ldots}+\delta_{\ldots, 1}+(\alpha \delta)_{\mathrm{i}, 1}+\pi_{\ldots, \mathrm{s}}+(\alpha \pi)_{\mathrm{i}, 1 \mathrm{~s}}+\bar{\varepsilon}_{\mathrm{i}, 1 \mathrm{~s}}$

$\overline{\mathrm{X}}_{. \mathrm{j} 1 \mathrm{~s}} \quad \mu_{\ldots}+\beta_{. \mathrm{j}}+\delta_{\ldots 1}+(\beta \delta)_{\mathrm{j} 1}+\pi_{\ldots \mathrm{s}}+(\beta \pi)_{. \mathrm{j} 1 \mathrm{~s}}+\bar{\varepsilon}_{\mathrm{j} 1 \mathrm{~s}}$

$\mathrm{X}_{\mathrm{ij} 1 \mathrm{~h}} \quad \mu \ldots+\alpha_{\mathrm{i}} \ldots+\beta_{\mathrm{j}} \ldots+\delta_{\ldots 1}+(\alpha \beta)_{\mathrm{ij}} \ldots+(\alpha \delta)_{\mathrm{i}, 1}+(\beta \delta)_{\mathrm{j} 1}+(\alpha \beta \delta)_{\mathrm{ijl}}+\pi_{\ldots \mathrm{k}}+(\alpha \pi)_{\mathrm{i}, \mathrm{k}}+(\beta \pi)_{\mathrm{j} 1 \mathrm{~s}}$

$$
+(\alpha \beta \pi)_{i j l \downarrow}+\varepsilon_{i j l \varsigma}
$$




\begin{tabular}{|c|c|}
\hline$\overline{\bar{X}}_{i, 2 s}$ & $\mu \ldots+a_{i} \ldots+\delta \ldots+(\alpha \delta)_{i, 2}+\pi_{i .2 s}+(\alpha \pi)_{i .2 s}+\xi_{1.2 s}$ \\
\hline $\mathrm{X}_{\mathrm{ij2s}}$ & $\begin{array}{l}\mu \ldots+\alpha_{\mathrm{i}} \ldots+\beta_{. \mathrm{j} . .}+\delta_{.2 .}+(\alpha \beta)_{\mathrm{ij} .}+(\alpha \delta)_{\mathrm{i} .2 .}+(\beta \delta)_{. \mathrm{j} 2}+(\alpha \beta \delta)_{\mathrm{ij} 2}+\pi_{\mathrm{i} .2 \mathrm{~s}}+(\alpha \pi)_{\mathrm{i} .2 \mathrm{~s}}+(\beta \pi)_{\mathrm{ij} 2 \mathrm{~s}} \\
+(\alpha \beta \pi)_{\mathrm{ij} 2 \mathrm{~s}}+\varepsilon_{\mathrm{ij} 2 \mathrm{~s}}\end{array}$ \\
\hline
\end{tabular}

NOTE-Means with expressions unchanged from Case 1 are not listed.

Table 6

Expected Values of Mean Squares for Case 2

\begin{tabular}{|c|c|c|}
\hline Source of Variation & Expected Value of Mean Square & Denominator \\
\hline $\mathbf{A}$ & $2 \mathrm{JnS} \mathrm{S}_{\mathrm{A}}^{2}+\frac{\mathrm{J}}{2} \sigma_{\mathrm{s}}^{2}+\frac{\mathrm{J}(2 \mathrm{I}-1)}{2 \mathrm{I}} \mathrm{S}_{\mathrm{As}}^{2}+\sigma_{\varepsilon}^{2}$ & $2 a$ \\
\hline B & $2 \operatorname{InS}_{\mathrm{B}}^{2}+\frac{(I+1)}{2} S_{\mathrm{Bs}}^{2}+\frac{(I-1)}{2 I} S_{A B s}^{2}+\sigma_{\varepsilon}^{2}$ & $2 b$ \\
\hline $\mathrm{A} \times \mathrm{B}$ & $2 \mathrm{nS}_{\mathrm{AB}}^{2}+\frac{1}{2} \mathrm{~S}_{\mathrm{Bs}}^{2}+\frac{(2 \mathrm{I}-1)}{2 \mathrm{I}} \mathrm{S}_{\mathrm{ABs}}^{2}+\sigma_{\varepsilon}^{2}$ & $2 c$ \\
\hline D & $\mathrm{IJnS}_{\mathrm{D}}^{2}+\frac{\mathrm{J}(\mathrm{I}+1)}{2} \sigma_{\mathrm{S}}^{2}+\frac{\mathrm{J}(\mathrm{I}-1)}{2 \mathrm{I}} \mathrm{S}_{\mathrm{As}}^{2}+\sigma_{\mathrm{C}}^{2}$ & $2 d$ \\
\hline$A \times D$ & $\mathrm{JnS}_{\mathrm{AD}}^{2}+\frac{\mathrm{J}}{2} \sigma_{\mathrm{S}}^{2}+\frac{\mathrm{J}(2 \mathrm{I}-1)}{2 \mathrm{I}} \mathrm{S}_{\mathrm{AS}}^{2}+\sigma_{\varepsilon}^{2}$ & $2 a$ \\
\hline$B \times D$ & $\operatorname{InS}_{\mathrm{BD}}^{2}+\frac{(I+1)}{2} S_{\mathrm{Bs}}^{2}+\frac{(I-1)}{2 I} S_{A B s}^{2}+\sigma_{\varepsilon}^{2}$ & $2 b$ \\
\hline$A \times B \times D$ & $\mathrm{nS}_{\mathrm{ABD}}^{2}+\frac{1}{2} \mathrm{~S}_{\mathrm{BS}}^{2}+\frac{(2 \mathrm{I}-1)}{2 \mathrm{I}} \mathrm{S}_{\mathrm{ABs}}^{2}+\sigma_{\varepsilon}^{2}$ & $2 c$ \\
\hline$s\left(D_{1}\right)$ & $\mathrm{IJJ} \mathrm{s}_{\mathrm{s}}^{2}+o_{\varepsilon}^{2}$ & \\
\hline $\operatorname{Axs}\left(D_{1}\right)$ & $\mathrm{JS}_{\mathrm{As}}^{2}+\sigma_{\varepsilon}^{2}$ & \\
\hline $\operatorname{Bxs}\left(D_{1}\right)$ & $\mathrm{IS}_{\mathrm{Bs}}^{2}+\mathrm{O}_{\varepsilon}^{2}$ & \\
\hline $\operatorname{AxBxs}\left(D_{1}\right)$ & $\mathrm{S}_{\mathrm{ABs}}^{2}+\sigma_{\varepsilon}^{2}$ & \\
\hline $\mathrm{s} / \mathrm{A}\left(\mathrm{D}_{2}\right)$ & $\mathrm{J} \sigma_{\mathrm{s}}^{2}+\frac{\mathrm{J}(\mathrm{I}-1)}{\mathrm{I}} \mathrm{S}_{\mathrm{As}}^{2}+\sigma_{\varepsilon}^{2}$ & \\
\hline $\mathrm{Bxs} / \mathrm{A}\left(\mathrm{D}_{2}\right)$ & $S_{B s}^{2}+\frac{(I-1)}{I} S_{A B s}^{2}+\sigma_{\varepsilon}^{2}$ & \\
\hline
\end{tabular}

needed to test all effects. Each is determined by taking the arithmetic mean of two mean squares. First,

$$
\text { Denominator } 2 \mathrm{a}=\left[\mathrm{MS}_{\mathrm{Axs}\left(\mathrm{D}_{1}\right)}+\mathrm{MS}_{\mathrm{s} / \mathrm{A}\left(\mathrm{D}_{2}\right)}\right] / 2
$$

is to be used to test the effect of $A$ and the $A \times D$ interaction. To test the effect of $B$ and the $B \times D$ interaction, one uses

Denominator $2 b=\left[M_{\mathrm{Bxs}\left(\mathrm{D}_{1}\right)}+\mathrm{MS}_{\mathrm{Bxs} / \mathrm{A}\left(\mathrm{D}_{2}\right)}\right] / 2$.
To test the $A \times B$ and the $A \times B \times D$ interactions, one uses

Denominator $2 \mathrm{c}=\left[\mathrm{MS}_{\mathrm{AxBxs}\left(\mathrm{D}_{1}\right)}+\mathrm{MS}{\mathrm{Bxs} / \mathrm{A}\left(\mathrm{D}_{2}\right)}\right] / 2$. Finally,

Denominator $2 \mathrm{~d}=\left[\mathrm{MS}_{\mathrm{S}_{\left(\mathrm{D}_{1}\right)}}+\mathrm{MS}_{\mathrm{S} / \mathrm{A}\left(\mathrm{D}_{2}\right)}\right] / 2$

is used to test the main effect of $\mathrm{D}$. The last column of Table 6 indicates the different denominators to be used. The number of degrees of freedom associated 
with each of the denominators can again be estimated using Satterthwaite's (1946) formula.

\section{Case 3: AwBw-AbBb}

In the last case, the within-subjects between-subjects comparison is of interest for both substantive independent variables. The experiment consists of $\mathrm{IJ}+1$ independent groups requiring $n(I J+1)$ subjects. At $D_{1}$ there is a single group of subjects. Each of the subjects in this group receives all of the $I J$ treatments stemming from the $I \times J$ crossing of variables $A$ and B. This is exactly equivalent to design $D_{1}$ in the last case, $A w B w-A b B w$. At $D_{2}$ there are IJ independent groups. Each of these groups receives one of the $\mathrm{IJ}$ treatments resulting from the $\mathrm{I} \times \mathrm{J}$ crossing of variables $\mathrm{A}$ and $\mathrm{B}$. This D2 is the same D2 design as in the first case, $\mathrm{AwBb}-\mathrm{AbBb}$.

The model for Case 3 has

$$
\begin{aligned}
\mathrm{X}_{\mathrm{ijks}}= & \mu \ldots+\alpha_{\mathrm{i}} \ldots+\beta_{. \mathrm{j} . .}+\delta_{. \mathrm{k}} \\
& +(\alpha \beta)_{\mathrm{ij}} . .+(\alpha \delta)_{\mathrm{i} . \mathrm{k} .}+(\beta \delta)_{. j \mathrm{k} .} \\
& +(\alpha \beta \delta)_{\mathrm{ijk} .}+\pi_{\mathrm{ijks}}+(\alpha \pi)_{\mathrm{ijks}}+(\beta \pi)_{\mathrm{ijks}} \\
& +(\alpha \beta \pi)_{\mathrm{ijks}}+\varepsilon_{\mathrm{ijks}} .
\end{aligned}
$$

The terms here are the same as those for Case 2, except that the $\pi$ and $(\alpha \pi)$ terms are differentiated according to $\mathrm{j}$ in this model; in the Case 2 model the $\mathrm{j}$ subscript on these two terms can be replaced by a dot. The normal analysis of variance assumptions are modified here by differentiating some terms between the two design types. In $D_{1}$

$$
\sum_{\mathrm{i}}(\alpha \pi)_{\mathrm{i} .1 \mathrm{~s}}=\sum_{\mathrm{j}}(\beta \pi)_{. j 1 \mathrm{~s}}=\sum_{\mathrm{i}}(\alpha \beta \pi)_{\mathrm{ij} 1 \mathrm{~s}}=\sum_{\mathrm{j}}(\alpha \beta \pi)_{\mathrm{ij} 1 \mathrm{~s}}=0,
$$

while only the expected values of those terms equal zero in $\mathrm{D}_{2}$ :

$$
\mathrm{E}(\alpha \pi)_{\mathrm{ij} 2 \mathrm{~s}}=\mathrm{E}(\beta \pi)_{\mathrm{ij} 2 \mathrm{~s}}=\mathrm{E}(\alpha \beta \pi)_{\mathrm{ij} 2 \mathrm{~s}}=0 .
$$

The partitioning of the total sum of squares is the same for Cases 2 and 3, with the exception that the last two terms in the Case 2 partitioning, $s / A\left(D_{2}\right)$ and $B x s / A\left(D_{2}\right)$, are replaced by a single source of variation, subjects $/ \mathrm{AB}\left(\mathrm{D}_{2}\right.$ only), [s/AB($\left.\left(D_{2}\right)\right]$, which has degrees of freedom and sum of squares:

$$
\begin{gathered}
\mathrm{df}=\mathrm{IJ}(\mathrm{n}-1) \\
\mathrm{SS}=\sum_{\mathrm{i} j \mathrm{j} s} \sum_{\mathrm{ij} 2 \mathrm{~s}}-\overline{\mathrm{X}}_{\mathrm{ij} 2 .)^{2} .}
\end{gathered}
$$

Table 7 gives the expression of the means appearing in the sums of squares in terms of the model effects. Means whose expressions are unchanged from Case 2 do not appear in the table. Furthermore, because the changes in the expressions for $\overline{\mathrm{X}}_{\mathrm{j}}$... $\overline{\mathrm{X}}_{\mathrm{ij} . .,}, \overline{\mathrm{X}}_{. \mathrm{j} 2 .}, \overline{\mathrm{X}}_{\mathrm{ij} 2 .}$, and $\mathrm{X}_{\mathrm{ij} 2 \mathrm{~s}}$ are trivial, the means do not appear in the table. The only changes that occur for these means is that $\bar{\pi} . .2,(\overline{\alpha \vec{\pi}}) \ldots 2, \bar{\pi}_{\mathrm{i}} .2$. , $(\overline{\alpha \pi})_{i} .2, \bar{\pi}_{i} .2 s$, and $(\alpha \pi)_{i .2 s}$ appearing in Case 2 expressions are replaced by $\bar{\pi}_{. j 2},(\overline{\alpha \pi}) . j 2, \bar{\pi}_{\mathrm{ij} 2}$, $(\overrightarrow{\alpha \pi})_{i j 2}, \pi_{i j 2 s}$, ar,d $(\alpha \pi)_{i j 2 s}$, respectively. Again, we must note differences in the number of independent scores on which some means in the two design types are based. In $D_{1},(\bar{\beta} \pi)$, j1 . and $(\beta \pi)$, j1s are based on $\mathrm{n}$ scores and one score, respectively, while their counterparts in $\mathrm{D}_{2}$ are based on I times that many scores; $(\overline{\alpha \pi})_{i}, 1$ and $(\alpha \pi)_{i} .1 \mathrm{~s}$ are based on $\mathrm{n}$ and one score(s), respectively, while their counterparts are based on $\mathrm{J}$ times as many scores; $\bar{\pi}_{\ldots} .1$ and $\pi_{\text {. . 1s }}$ are based on $\mathrm{n}$ and one score(s), respectively, and their counterparts are based on IJ times as many scores.

Table 8 shows the expected values of the mean squares associated with each source of variation. Four different denominators are required for hypothesis tests; each will be an arithmetic mean of two mean squares. To test the $\mathrm{A}$ and $\mathrm{A} \times \mathrm{D}$ effects, we need

$$
\text { Denominator } 3 \mathrm{a}=\left[\mathrm{MS}_{\mathrm{Axs}\left(\mathrm{D}_{1}\right)}+\mathrm{MS}_{\mathrm{S} / \mathrm{AB}\left(\mathrm{D}_{2}\right)}\right] / 2 \text {. }
$$

To test the $B$ and $B \times D$ effects, we use

$$
\text { Denominator } 3 \mathrm{~b}=\left[\mathrm{MS}_{\mathrm{Bxs}\left(\mathrm{D}_{1}\right)}+\mathrm{MS}{\mathrm{S} / \mathrm{AB}\left(\mathrm{D}_{2}\right)}\right] / 2 \text {. }
$$

The $\mathbf{A} \times \mathbf{B}$ and $\mathbf{A} \times \mathbf{B} \times \mathrm{D}$ interactions are tested with

Table 7

Means Expressed in Terms of Model Effects: Case 3

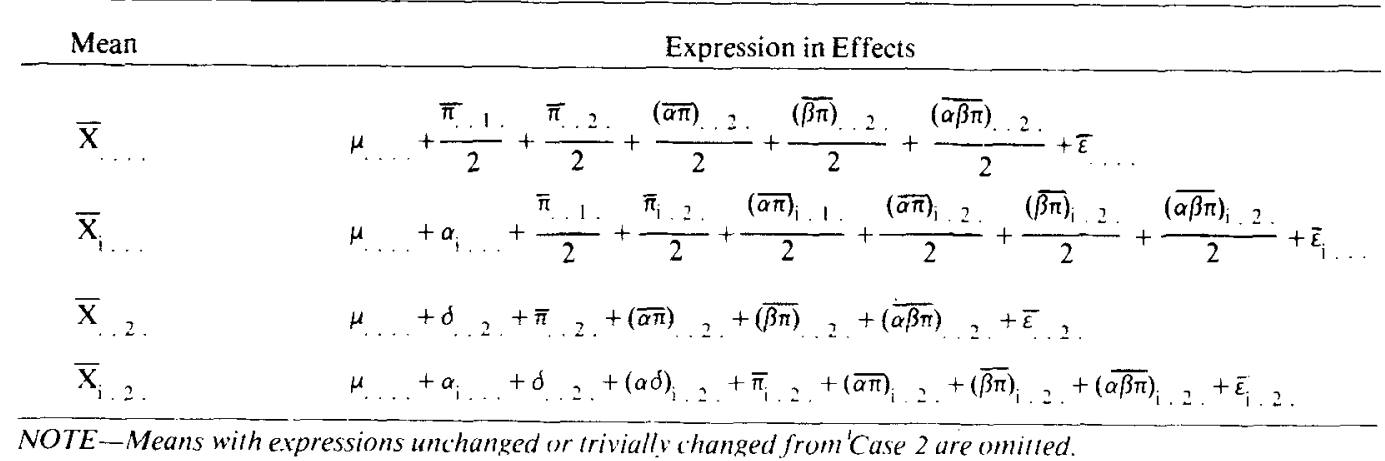


Table 8

Expected Values of Mean Squares for Case 3

\begin{tabular}{|c|c|c|}
\hline Source of Variation & Expected Value of Mean Square & Denominator \\
\hline A & $2 \mathrm{JnS} \mathrm{A}_{\mathrm{A}}^{2}+\frac{1}{2} \mathrm{D}_{\mathrm{S}}^{2}+\frac{(\mathrm{IJ}+\mathrm{I}-1)}{2 \mathrm{I}} \mathrm{S}_{\mathrm{AS}}^{2}+\frac{(\mathrm{J}-1)}{2 \mathrm{~J}} \mathrm{~S}_{\mathrm{BS}}^{2}+\frac{(\mathrm{IJ}-\mathrm{I}-\mathrm{J}+1)}{2 \mathrm{IJ}} \mathrm{S}_{\mathrm{ABS}}^{2}+\sigma_{\varepsilon}^{2}$ & $3 a$ \\
\hline B & $2 \ln S_{B}^{2}+\frac{1}{2} \sigma_{s}^{2}+\frac{(I-1)}{2 I} S_{A s}^{2}+\frac{(I J+J-1)}{2 J} S_{B s}^{2}+\frac{(I J-I-J+1)}{2 I J} S_{A B s}^{2}+\sigma_{\varepsilon}^{2}$ & $3 b$ \\
\hline $\mathbf{A} \times \mathbf{B}$ & $2 n S_{A B}^{2}+\frac{1}{2} a_{s}^{2}+\frac{(I-1)}{2 I} S_{A s}^{2}+\frac{(J-1)}{2 J} S_{B s}^{2}+\frac{(2 I J-I-J+1)}{2 I J} S_{A B s}^{2}+\sigma_{\varepsilon}^{2}$ & $3 c$ \\
\hline D & $\mathrm{IJnS}_{\mathrm{D}}^{2}+\frac{(\mathrm{IJ}+1)}{2} \sigma_{\mathrm{S}}^{2}+\frac{(\mathrm{I}-1)}{2 \mathrm{I}} \mathrm{S}_{\mathrm{AS}}^{2}+\frac{(\mathrm{J}-1)}{2 \mathrm{~J}} \mathrm{~S}_{\mathrm{BS}}^{2}+\frac{(\mathrm{IJ}-\mathrm{I}-\mathrm{J}+1)}{2 \mathrm{IJ}} \mathrm{S}_{\mathrm{ABS}}^{2}+\sigma_{\varepsilon}^{2}$ & 3d \\
\hline $\mathbf{A} \times \mathbf{D}$ & $\mathrm{JnS}_{\mathrm{AD}}^{2}+\frac{1}{2} \mathrm{o}_{\mathrm{s}}^{2}+\frac{(\mathrm{IJ}+\mathrm{I}-1)}{2 \mathrm{I}} \mathrm{S}_{\mathrm{As}}^{2}+\frac{(\mathrm{J}-1)}{2 \mathrm{~J}} \mathrm{~S}_{\mathrm{Bs}}^{2}+\frac{(\mathrm{IJ}-\mathrm{I}-\mathrm{J}+1)}{2 \mathrm{IJ}} \mathrm{S}_{\mathrm{ABs}}^{2}+o_{\mathrm{E}}^{2}$ & $3 a$ \\
\hline$B \times D$ & $\ln S_{B D}^{2}+\frac{1}{2} \sigma_{s}^{2}+\frac{(I-1)}{2 I} S_{A s}^{2}+\frac{(I J+J-1)}{2 J} S_{B s}^{2}+\frac{(I J-I-J+1)}{2 I J} S_{A B s}^{2}+\sigma_{\varepsilon}^{2}$ & $3 b$ \\
\hline $\mathbf{A} \times \mathbf{B} \times \mathbf{D}$ & $\mathrm{nS}_{\mathrm{ABD}}^{2}+\frac{1}{2} \sigma_{\mathrm{s}}^{2}+\frac{(I-1)}{2 I} \mathrm{~S}_{\mathrm{As}}^{2}+\frac{(\mathrm{J}-1)}{2 \mathrm{~J}} \mathrm{~S}_{\mathrm{BS}}^{2}+\frac{(2 \mathrm{~J}-\mathrm{I}-\mathrm{J}+1)}{2 \mathrm{~J} J} \mathrm{~S}_{\mathrm{ABS}}^{2}+o_{\mathrm{E}}^{2}$ & $3 c$ \\
\hline$s\left(D_{l}\right)$ & $1 \mathrm{IJ} \sigma_{s}^{2}+\sigma_{\varepsilon}^{2}$ & \\
\hline $\operatorname{Axs}\left(D_{1}\right)$ & $\mathrm{JS}_{\mathrm{As}}^{2}+\sigma_{\varepsilon}^{2}$ & \\
\hline $\operatorname{Bxs}\left(D_{1}\right)$ & $\mathrm{IS}_{\mathrm{Bs}}^{2}+o_{\varepsilon}^{2}$ & \\
\hline $\operatorname{AxBxs}\left(D_{1}\right)$ & $\mathrm{S}_{\mathrm{ABs}}^{2}+\sigma_{\varepsilon}^{2}$ & \\
\hline $\mathrm{s} / \mathrm{AB}\left(\mathrm{D}_{2}\right)$ & $\sigma_{\mathrm{S}}^{2}+\frac{(\mathrm{I}-1)}{\mathrm{I}} \mathrm{S}_{\mathrm{AS}}^{2}+\frac{(\mathrm{J}-1)}{\mathrm{J}} \mathrm{S}_{\mathrm{BS}}^{2}+\frac{(\mathrm{IJ}-\mathrm{I}-\mathrm{J}+1)}{\mathrm{IJ}} \mathrm{S}_{\mathrm{ABs}}^{2}+\sigma_{\varepsilon}^{2}$ & \\
\hline
\end{tabular}

Denominator $3 \mathrm{c}=\left[\mathrm{MS}_{\mathrm{AxBxs}\left(\mathrm{D}_{1}\right)}+\mathrm{MS}_{\mathrm{s} / \mathrm{AB}\left(\mathrm{D}_{2}\right)}\right] / 2$.

Finally, the effect of variable $D$ is tested using

$$
\text { Denominator } 3 \mathrm{~d}=\left[\mathrm{MS}_{\mathrm{s}\left(\mathrm{D}_{1}\right)}+\mathrm{MS}_{\mathrm{S} / \mathrm{AB}\left(\mathrm{D}_{2}\right)}\right] / 2 \text {. }
$$

The final column of Table 3 indicates the denominators to be used to test the effect of the various sources of variation. Satterthwaite's formula for estimating the number of degrees of freedom associated with each of the denominators is to be employed.

\section{A GENERAL RULE}

Although the expected values of mean squares differ for the three cases, a general rule emerges for determining the appropriate linear combination of mean squares for the testing of a specific effect. (1) For the test of any effect due to substantive independent variables $(A, B, A \times B)$, one obtains the arithmetic mean of the mean square that would be the appropriate denominator were the entire experiment done as $D_{1}$ and the mean square that would be the appropriate denominator were the entire experiment done as $D_{2}$. (2) The denominator for testing the main effect of $D$ is the arithmetic mean of the two subjects-within-groups mean squares. (3) The de- nominator for the test of any interaction with $D$ $(A \times D, B \times D, A \times B \times D)$ is the same denominator as that employed for the test of the effect interacting with $D(A, B$, and $A \times B$, respectively). From the fact that an interaction between two variables is not intrinsically different from a main effect, it follows that the rule need not be restricted to experiments with two substantive independent variables. The rule can be used for experiments of any size.

\section{REFERENCES}

ERLEBACher, A. Design and analysis of experiments contrasting the within- and between-subjects manipulation of the independent variable. Psychological Bulletin, 1977, 84, 212-219.

SATTERTHWAITE, F. E. An approximate distribution of estimates of variance components. Psychometrics Bulletin, 1946, 2, 110-114.

\section{NOTE}

1. The $D_{1}$ and $D_{2}$ designation differs from usage in the earlier paper. In that paper, the symbols W (for "within") and B (for "between") were used to designate the two levels of D. Since here neither level of $\mathrm{D}$ represents a purely within- or between-subjects design, the earlier symbols become inappropriate. In this paper, the letter $B$ is used to designate one of the two substantive independent variables.

(Received for publication March 21, 1978; revision accepted June $28,1978$. ) 